\title{
Mechanical behavior of architectured photosensitive silicone membranes: experimental data and numerical analysis.
}

\author{
G. Machado ${ }^{\mathrm{a}, \mathrm{b}}$, A. Stricher ${ }^{\mathrm{c}}$, G. Chagnon ${ }^{*, a, b}$, D. Favier ${ }^{\mathrm{a}, \mathrm{b}}$ \\ ${ }^{a}$ Université de Grenoble Alpes, TIMC-IMAG, F-38000 Grenoble, France. \\ ${ }^{b}$ CNRS, TIMC-IMAG, F-38000 Grenoble, France. \\ ${ }^{c}$ MATEIS, INSA Lyon 8 CNRS, UMR5510, Villeurbanne, France
}

\begin{abstract}
The aim of this paper is to provide an experimental and modeling study of an architectured photosensitive silicone membrane. Mechanical properties are dependent of UV doses used to alter the local cross-link density, resulting in a direct effect on the macroscopic mechanical behavior. A series of mechanical tests were carried out to characterize the mechanical behavior of each phase. Results are presented for various types of loading, including uniaxial, planar and equibiaxial loading cases, for the UV irradiated and the non-irradiated material. Using the bulge test with an architectured sample, the global stretchability with minimum boundary conditions perturbations was investigated. To further explore the unusual properties offered by silicone graded membranes, finite element analysis of graded architecture was performed to try to predict the stress-strain response in the bulge test. Soft-to-hard transition is tested and the macroscopic influence of interface was observed.
\end{abstract}

Key words: architectured membranes; photosensitive silicone; biocompatible silicone; bulge test; bi-material hyperelastic solid;

\section{Introduction}

Facing increasing demands for multifunctional solutions, architectured materials take an increasingly important place in many applications in order to design specific mechanical properties for a given purpose. Very often the function is not provided by the local property only (grain size, precipitation, polymer chain design and interchain bonding, state of crystallization), but by the interplay between the shape, the properties, and possible association of materials [1].

\footnotetext{
${ }^{*}$ Corresponding author

Email addresses: guilherme.machado@imag.fr (G. Machado), arthur.stricher@insa-lyon.fr (A. Stricher), gregory.chagnon@imag.fr (G. Chagnon), denis.favier@imag.fr (D. Favier)
} 
For instance, some polymers are architectured due to their chain design [2]. Usually, this strategy operates at scales between $1 \mathrm{~nm}$ and $10 \mu \mathrm{m}$. Architectured silicone materials can also be composed with an association of materials (also called hybrid material) for example fiberreinforced [3] or NiTi-reinforced membranes [4]. Even if such composites are very good candidates for biomimetic membranes, this solution involves the integration of different synthetic materials often associated with local mismatches of mechanical properties and adhesion difficulties. Local mismatches may cause excessive stress concentrations within the structure and thus premature failure of the composite upon stretching. Thus, one of the challenges of the architectured material is in ensuring efficient stress transfer and in avoiding local failure between regions of different mechanical properties.

Other materials can be considered as architectured because of their geometry. [5] and [6] developed crenelated membranes with an unfilled and filled silicone rubber. The main advantage of these membranes is that they present an anisotropic behavior without any interface in the material. Indeed, the crenels and their orientations allow to induce and control the anisotropy, but this fact is limited by the out-plane geometry and the process to obtain the reinforced membrane.

This paper focuses on the mechanical behavior of architectured silicone membranes where the membrane architecture is controlled by the in-plane intrinsic properties but also by a desired topology at scale between the microstructure and the application. The concept is to create a heterogeneous material with locally tuned mechanical properties by changing the local crosslink density. The approach can be exploited, for example, to create bioinspired membranes that mimic anisotropic structural properties of soft tissues. In this context, Section 2 presents all precautions concerning the experimental mechanical testing procedures and strain field measurements techniques. Experimental data and analyzes are presented into two parts. First, in Section 3, the three deformations modes (uniaxial, planar and equibiaxial tensile tests) for each phase are independently tested. Second, in Section 4, the bulge test of graded membrane containing two phases. In Section 5, a finite element analysis (FEA) is carried out using a hyperelastic model fitted simultaneously on the three previous tensile tests. Then, the numerical model was used to try to predict the bimaterial and results are discussed. Finally, Section 6 contains some concluding remarks and outlines some future perspectives. 


\section{Testing procedures background and strain field measurements techniques}

A series of mechanical tests were carried out to characterize the silicone mechanical behavior in its soft and hard phases. First, for the three deformations modes: uniaxial, planar (pure shear) and equibiaxial tensile tests; second, for the bulge test of graded membrane containing two phases.

\subsection{Preparation of the silicone specimens}

Samples were prepared in the IMP laboratory (Ingénierie des Matériaux Polymères - Villeurbanne, France), using the polydimethylsiloxane (PDMS) elastomer in addition of an UV-sensitive photoinhibitor. The membrane was selectively exposed to UV radiation then the cross-linking of the UV exposed elastomer is inhibited, leading to a softer material than the unexposed zone. From this point forward, the soft phase denotes the UV exposed material and the hard phase the unexposed one.

\subsection{In-plane tests}

In-plane quasi-static experiments were conducted on a Gabo Explorer testing machine with $\pm 25 \mathrm{~N}$ and $\pm 500 \mathrm{~N}$ load cells for uniaxial tension and planar tension respectively. A 2D digital image correlation system (DIC) was used during the test to obtain 2D fields at the surface of plane specimens. The commercial VIC-2D 2009 software package from Correlated solutions was used to acquire images. The images were recorded at $1 \mathrm{~Hz}$ with a Pike F-421B/C CCD camera with a sensor resolution of $C_{r}=7.4 \mu \mathrm{m} /$ pixel. The reason for this large sensor format is the goal to achieve high resolution images with low noise. The $50 \mathrm{~mm}$ camera lens was set to $f / 22$ using a 50 pixels extension ring. Grayscale 8 bit images were captured using a full scan of 2048 pixels $\times 2048$ pixels. After all, a cross-correlation function was used and displacement vectors were calculated by correlating square facets (or subsets) of $f_{\text {size }}=21$ pixels and grid spacing $G_{s}=10$ pixels to carry out the correlation process for the undeformed and deformed images. To achieve a sub-pixel accuracy, optimized 8-tap splines were used for the gray value interpolation. As the optimization criteria for the subset matching, a zero-normalized squared difference was adopted, which is insensitive to offset and scale in lighting. For the weighting function, the Gaussian distribution was selected, as it provides the best compromise between 
spatial and displacement resolution [7]. In uniaxial tensile experiment, the spatial resolution (the physical size per pixel) was $S_{r}=15 \mu \mathrm{m}$. Likewise, for the planar tension $S_{r}=7 \mu \mathrm{m}$.

\subsection{Out-plane tests}

The bulge test was conducted in order to determinate an equibiaxial state of both phases and also tested the soft-hard bimaterial. A syringe driver was used and the internal pressure is measured by an AZ-8215 digital manometer. Seeing that the material is partially transparent, milk was used as hydrostatic fluid to increase the gray contrast for DIC and to avoid internal reflections. Inflation was sufficient slow to obtain a quasi-static load. Under the assumption of material isotropy over the circumferential direction, the principal directions of both stretch and stress tensors at each material particle are known ab initio to be the meridional and circumferential directions of the membrane surface. From this point forward, these directions will be denoted by the subscripts $m$ and $c$ respectively.

Assuming quasi-static motion, the equilibrium equations for a thin axisymmetric isotropic membrane, as adopted by Hill [8], can be expressed as

$$
\begin{aligned}
\sigma_{m} & =\frac{p}{2 h \kappa_{c}} \\
\sigma_{c} & =\frac{p}{2 h \kappa_{c}}\left(2-\frac{\kappa_{m}}{\kappa_{c}}\right)
\end{aligned}
$$

where $\left(\sigma_{m}, \sigma_{c}\right)$ are the meridional and circumferential stresses and $\left(\kappa_{m}, \kappa_{c}\right)$ are the meridional and circumferential curvatures. $h$ is the current thickness and $p$ is the time-dependent normal pressure acting uniformly $(d p / d R=0)$ over the radius $R$. As mentioned in [9] and [10] a remarkable consequence of membrane theory is that it admits equilibrium solutions without explicitly requiring a constitutive equation, since the equilibrium equations are derived directly by balancing forces of a deformed element shape. As a consequence, they are valid for all classes of in-plane isotropic materials.

Recently, Machado et al. [11] presented a methodology to compute the membrane curvature of the bulge test from 3D-DIC measurements. A very convenient calculation scheme was proposed based on the surface representation in curvilinear coordinates. From that scheme, the circumferential and meridional curvatures, and also the respective stresses, can be computed. In [11] authors presented an evaluation scheme for the bulge test based on the determination of the 
surface curvature tensor and the membrane stress tensor. With this method, the circumferential as well as the meridional stress can be determined at every stage and position of the specimen.

The commercial VIC-3D 7 software package from Correlated solutions was used to acquire images using two digital Pike cameras described in Section 2.3. Both cameras were set up at $D=150 \mathrm{~mm}$ distance and $35^{\circ}$ angle to the specimen using $28 \mathrm{~mm}$ focal length lenses opened at $f / 16$. Previous to the test, a good calibration of the 3D-DIC system is required. The following correlation options were chosen: 8-tap splines were used for the gray value interpolation, zeronormalized squared difference for the subset matching and the Gaussian distribution for the weighting function. Square facets of $f_{\text {size }}=15$ pixels and grid spacing $G_{s}=5$ pixels to carry out the correlation process for the undeformed and deformed images. The obtained spatial resolution is $S_{r}=15 \mu \mathrm{m}$.

Note that the spatial resolution $\left(S_{r}\right)$ of the discretized surface depends essentially on camera sensor resolution $\left(C_{r}\right)$ and on the choice of the grid spacing $\left(G_{s}\right)$ that defines the distance between the data points on the object. The grid spacing is the distance between the grid points in pixel. Thus, grid spacing limits the spatial resolution, as each grid point represents one single data point of the result. The facet size controls the area of the image that is used to track the displacement between images. The minimal facet size $\left(f_{\text {size }}\right)$ is limited by the size and roughness of the stochastic pattern on the object surface. Each facet must be large enough to ensure that there is a sufficiently distinctive pattern, with good contrast features, contained in the area-of-interest used for correlation.

\section{Soft and Hard phases: experimental results and analysis}

\subsection{Uniaxial tension test}

Uniaxial tensile tests were performed on small dog-bone shaped specimens. The samples had an initial gage length $l_{0}=12 \mathrm{~mm}$, width $w_{0}=4 \mathrm{~mm}$ and thickness $h_{0}=0.8 \mathrm{~mm}$. During the test, using an elongation rate of $\dot{\lambda}=3.0 \times 10^{-2} \mathrm{~s}^{-1}$, the nominal stress tensor $\mathbf{P}$ (First Piola-Kirchhoff stress tensor) is assumed to be homogeneous within the gauge region as well as the deformation gradient tensor F. Since the current thickness is not measured, the material is assumed to be incompressible, i.e., $\operatorname{det}(\mathbf{F})=1$. A cyclic loading-unloading test was realized for soft and hard phases, the curves are presented in Fig. 1. In the same figure, the first load 
of both phases are plotted. Different phenomena are highlighted, first a large stress-softening appears by comparing the two first loading at each strain level. A little hysteresis after the first cycle is observed. Moreover, few residual elongation is observed for both phases.

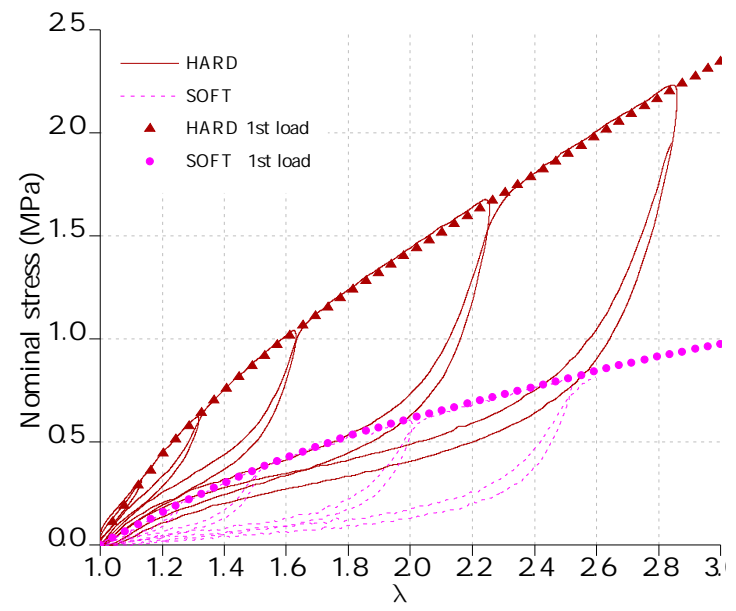

Figure 1: Nominal stress-strain curves resulting from cyclic loading-unloading tensile test at $\dot{\lambda}=3.0 \times 10^{-2} \mathrm{~s}^{-1}$ for soft and hard phases.

\subsection{Planar tension test}

The pure shear strain state was approached by performing planar tension test. The initial height $l_{0}$, the constant width $w_{0}$ and the thickness $h_{0}$ of samples were $4.5 \mathrm{~mm}, 40 \mathrm{~mm}$ and $0.8 \mathrm{~mm}$, respectively. The width of the specimen used for planar tension test must be at least ten times greater than its length. These dimensions have as objective to create an experiment where the specimen is constrained in the lateral direction such that all specimen thinning occurs in the thickness direction. A cyclic planar loading test was realized for both phases at $\dot{\lambda}=$ $1.0 \times 10^{-2} \mathrm{~s}^{-1}$. The results are presented in Fig. 2. Planar tensile response, likewise uniaxial traction, presents the same phenomena. For the soft phase, the maximum principal stretch experienced by the planar specimens are smaller if compared with uniaxial tensile test specimens. In general, this limitation lies in the fact that the planar tensile specimens must be constrained in the lateral direction without slipping. In this manner, the annoying premature tearing at the grips is observed. This is the major difficulty in planar tensile tests of thin specimens. 


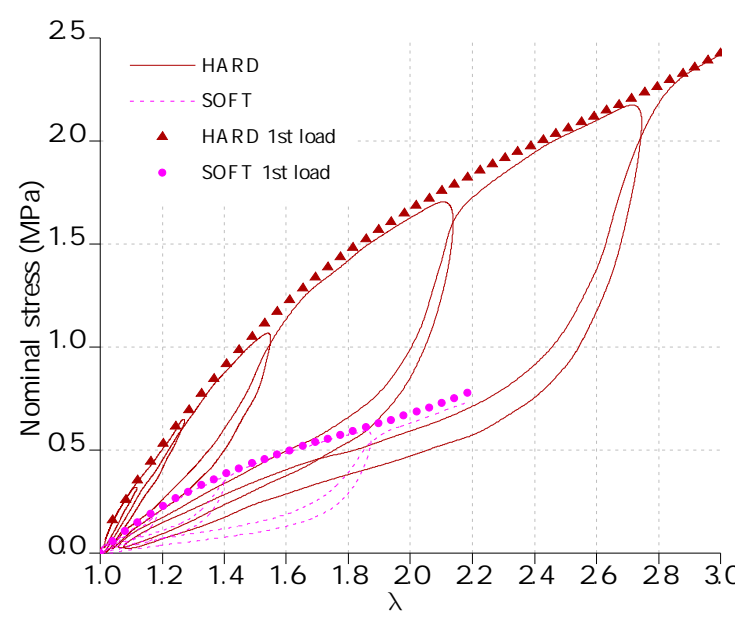

Figure 2: Nominal stress-strain curves resulting from cyclic loading-unloading planar tension test at $\dot{\lambda}=1.0 \times$ $10^{-2} \mathrm{~s}^{-1}$ for soft and hard phases.

\subsection{Equibiaxial tension using the bulge test}

The equibiaxial tension state is approached by the bulge test. Due to the axial-symmetry of the experimental configuration the equibiaxiality of the stress and strain is obtained at the top the inflated sample. The elongation rate was not controlled, but the pressure $p$ is slowly increased. The stress-strain curve for the central area are presented in Fig. 3 for a cyclic loading. The response are qualitatively similar to uniaxial loading with hysteresis and stress-softening.

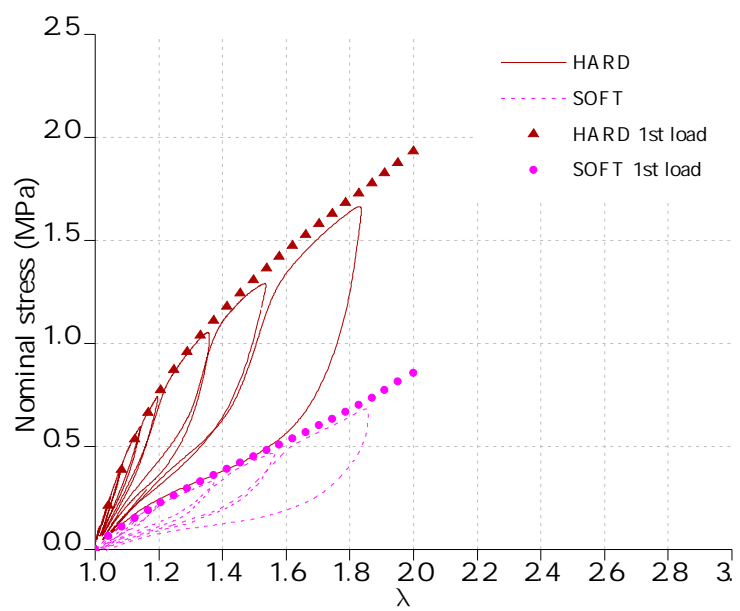

Figure 3: Nominal stress-strain curves resulting from cyclic loading-unloading equibiaxial test: (a) hard phase; (b) soft phase.

\subsection{Analysis}

Table 1 presents a comparative for soft and hard phases for the three different experimental load cases (uniaxial, plane shear and equibiaxial). It is easily determined from classical isotropic elasticity theory that, during quasi-linear stages, the ratio of the stress-strain slopes between uniaxial tension $(E)$ and equibiaxial tension $\left(E_{e}\right)$ is $E_{e} / E=1 /(1-\nu)$. The ratio $E_{e} / E$ was 
determined experimentally to be 2 for both phases. This is compatible with an incompressible material with Poisson's ratio $\nu=0.5$. The Young's modulus ratio between the two phases $\left(E_{H / S}\right)$ was about 3.5 for uniaxial and equibiaxial states and 2.5 for plane shear. At the beginning of loading, the stress ratio between phases at $\lambda=1.1$ of strain $\left(P_{S}^{10 \%}\right)$ are closer than stress ratio at $\lambda=2.0$. The mean stress ratio $\bar{P}$ calculates over all load history is kept practically constant for all loading cases.

Table 1: A comparative for soft and hard phases for the three different experimental loading cases.

\begin{tabular}{c|c|c|c|c|c}
\hline Parameter & & Unit & Uniaxial & Plane shear & Equibiaxial \\
\hline Hard phase elastic modulus & $E_{H}$ & $\mathrm{MPa}$ & 2.50 & 3.50 & 4.90 \\
Soft phase elastic modulus & $E_{S}$ & $\mathrm{MPa}$ & 0.70 & 1.40 & 1.40 \\
Elastic modulus ratio & $E_{H / S}$ & - & 3.57 & 2.50 & 3.50 \\
\hline Hard stress at $10 \%$ & $P_{H}^{10 \%}$ & $\mathrm{MPa}$ & 0.24 & 0.31 & 0.45 \\
Soft stress at $10 \%$ & $P_{S}^{10 \%}$ & $\mathrm{MPa}$ & 0.08 & 0.13 & 0.13 \\
\hline Hard stress at $100 \%$ & $P_{H}^{100 \%}$ & $\mathrm{MPa}$ & 1.43 & 1.67 & 1.93 \\
Soft stress at $100 \%$ & $P_{S}^{100 \%}$ & $\mathrm{MPa}$ & 0.61 & 0.68 & 0.85 \\
\hline Mean stress ratio & $\bar{P}$ & - & 2.44 & 2.44 & 2.90 \\
\hline
\end{tabular}

\section{Bulge test with soft-hard phase sample}

The specimen disk effective dimensions are $18.5 \mathrm{~mm}$ of radius and $0.4 \mathrm{~mm}$ of thickness. The UV-irradiated zone is concentric circle of $10 \mathrm{~mm}$ diameter. Cross-linking of the UV exposed elastomer is inhibited, leading to softer region than the surrounding unexposed part,as illustrated in Fig. 4.

The bulge test was chosen to test the bimaterial for two main reasons: stress-concentrations can be easily access the soft-hard interface is far from boundary conditions; each inflation state involves a heterogeneous stress-strain state which can be determined analytically. Having said that, bulge offers a valuable data for modeling benchmark. 

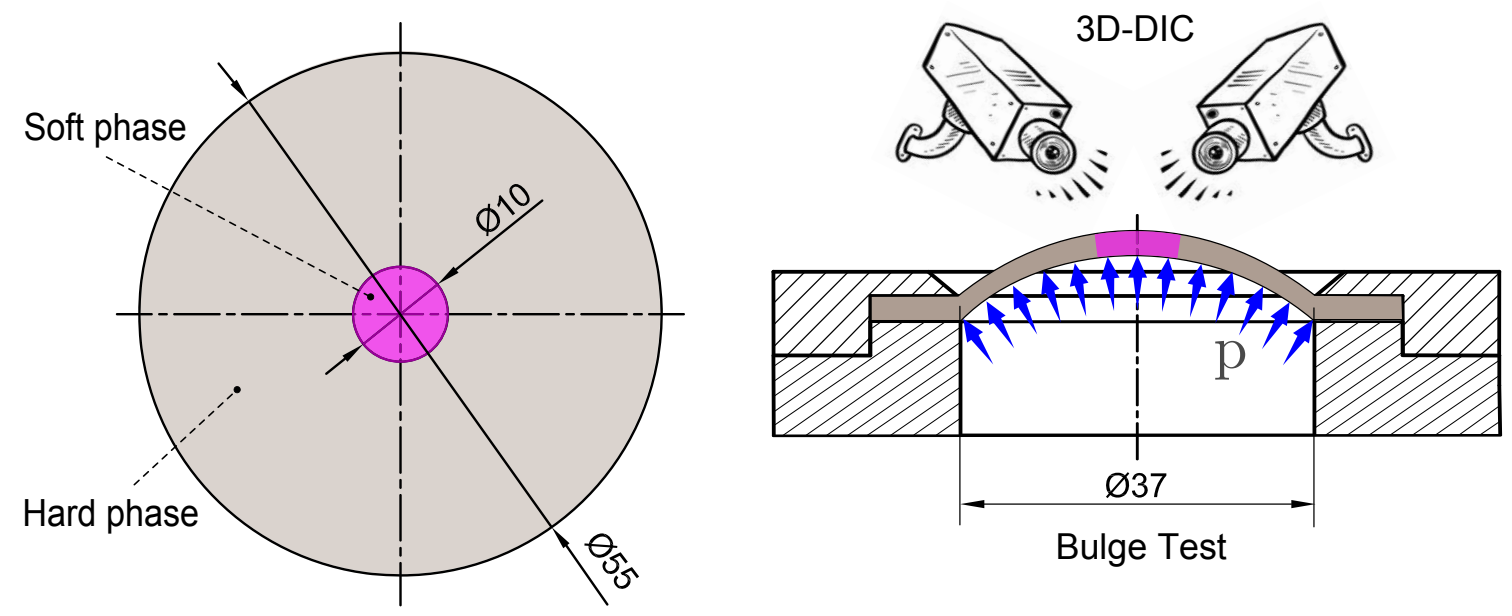

Figure 4: The photosensitive material sample and the bulge test configuration. (See online version for color figure.)

Fig. 5a shows the bulge test setup. The experimental image was superposed with the GreenLagrange major principal strain field for the maximal load pressure level $p=25 \mathrm{kPa}$ where $R$ is the normalized radius. It can be seen in the strain map that the isostrain contours are practically circular, indicating circumferential isotropy of the membrane. Plots of the deformed soft-hard membrane profiles for each of the seven inflation states can be found in Fig. 5b. The results presented in the following are mean values obtained over ten random meridians. The soft phase is in the interval $R=[0,0.27]$. The measured interval is represented by a colored box in plots. At the beginning of the loading, profiles resemble a generally smooth classical dome form. Increasing pressure, the deformed membrane develops a nipple geometry at the $R=[0,0.27]$ interval.

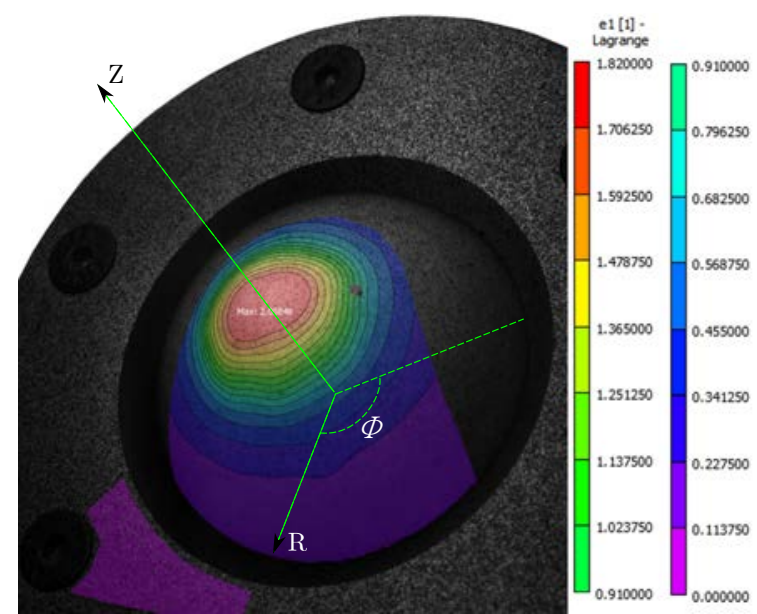

(a)

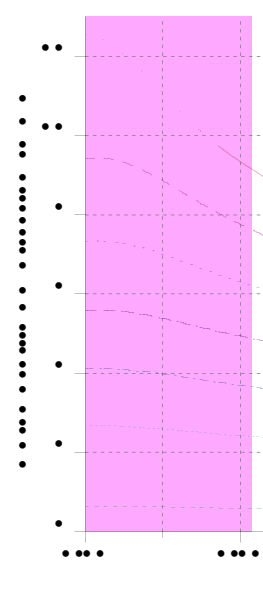

(b)

Figure 5: Bulge test setup using 3D-DIC technique. (a) Experimental image superposed with the Green-Lagrange major principal strain field (meridional strain); (b) Profiles of the inflated membrane composed by soft and hard phases for different pressure loads. (See online version for color figure.) 
Inflations were performed from $1 \mathrm{kPa}$ to a maximum pressure of $25 \mathrm{kPa}$, therefore, for clarity, three levels were chosen to present the results: 6,15 and $25 \mathrm{kPa}$. These inflations yielded principal stretches at the pole of about 1.16, 1.43 and 2.21 respectively. Fig. 6 presents principal stretches values $\left(\lambda_{m}, \lambda_{c}\right)$ obtained from 3D-DIC system. Save the pole $(R=0)$ and the clamped boundary $(R=1)$, all material points involve a heterogeneous strain state. As expected, the circumferential stretch $\lambda_{c}$ tends to one, i.e., a pure planar stretching behavior towards the clamped boundary $(R \rightarrow 1)$. However, most of the hard phase deformation is on the circumferential direction since $\lambda_{c}$ is less than 1.2 even for the maximal pressure level.

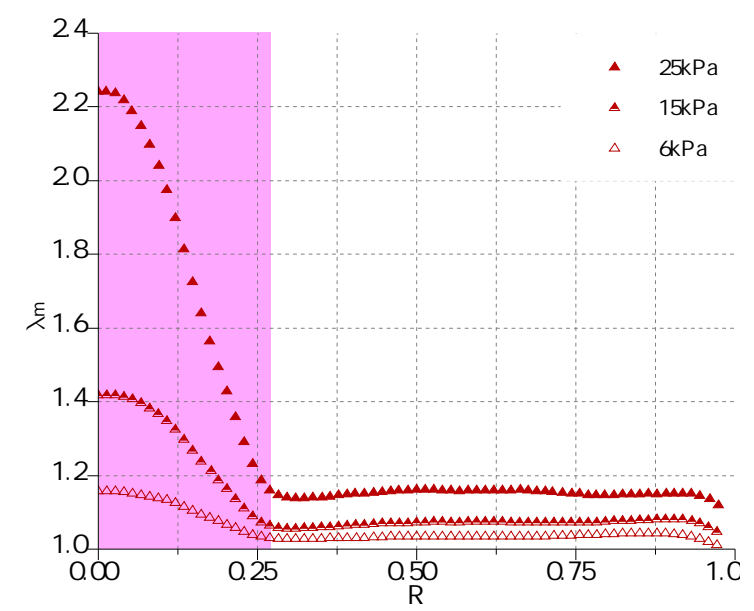

(a)

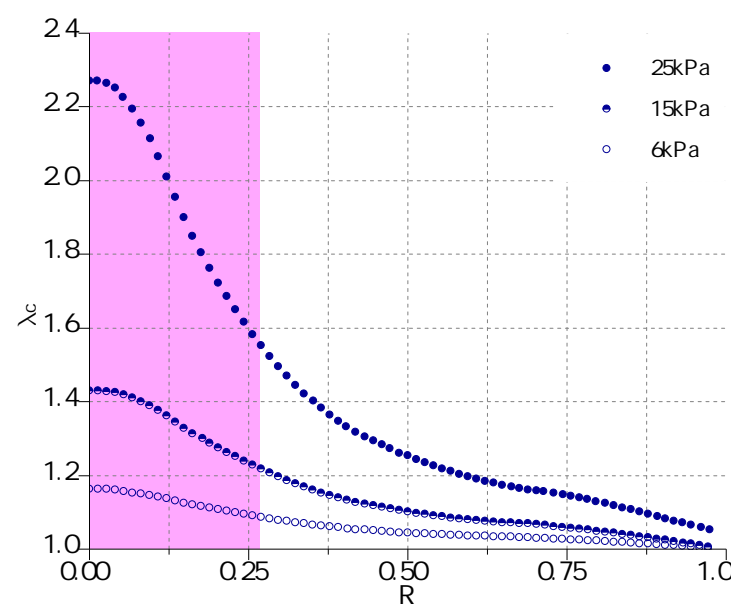

(b)

Figure 6: The strain distribution of a deformed foil vs. normalized radius of the circular membrane. (a) meridional direction $\lambda_{m}$; (b) circumferential direction $\lambda_{c}$.

Principal curvatures $\left(\kappa_{m}, \kappa_{c}\right)$ and principal stresses $\left(\sigma_{m}, \sigma_{c}\right)$ were computed as explained in [11]. Fig. 7 shows the experimental curves. With respect to principal curvature distributions, note that equibiaxial membrane deformations near the membrane pole $(R=0)$ are associated with an approximately spherical geometry, i.e., $\kappa_{m} \approx \kappa_{c}$. The small difference may be explained by the fact that the umbilical point may not lie exactly on the $Z$ direction axis. Note that for all pressure levels, the meridional curvature $\kappa_{m}$ presents an inflection point representing changes from convex to concave curvature on the soft-hard interface $(R=0.27)$.

With regard to the stress plots in Figs. $7 \mathrm{c}$ and $7 \mathrm{~d}$, the stress state can be assumed to be equibiaxial at the pole $(R=0)$. Both stresses, $\sigma_{m}$ and $\sigma_{c}$, experience an increasingly upward turn for $R \leqslant 0.27$ when pressure increases. Two inflections points can be observed around $R=0.27$ and $R=0.36$ in all directions for all pressure loads. 


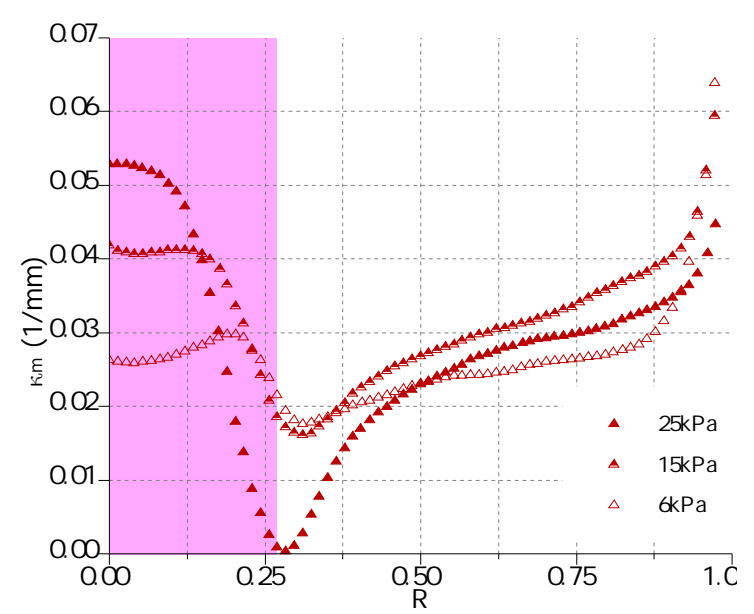

(a)

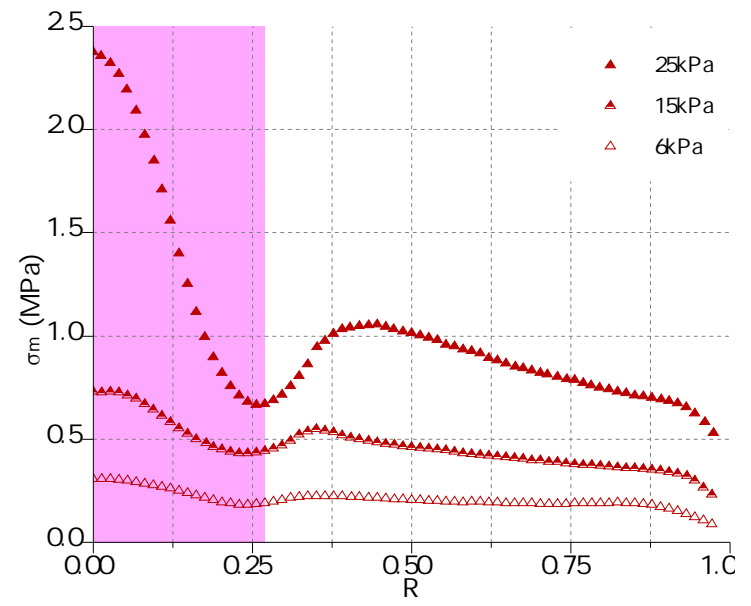

(c)

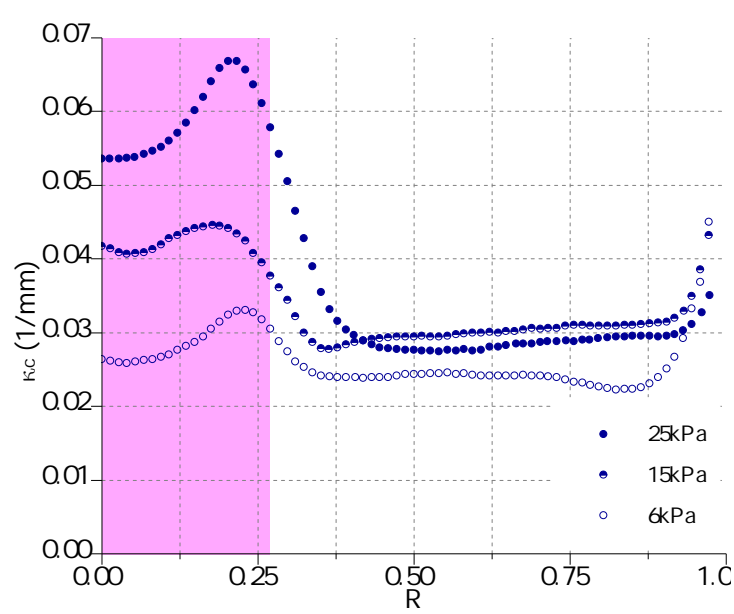

(b)

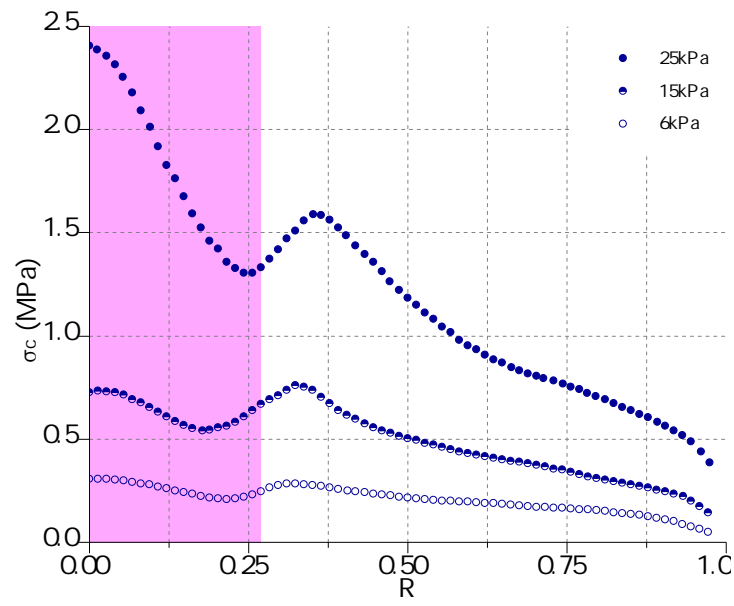

(d)

Figure 7: Distributions of principal direction of experimental fields corresponding to three different inflation states: (a) meridional curvature $\kappa_{m}$; (b) circumferential curvature $\kappa_{c}$; (c) meridional Cauchy stress $\sigma_{m}$; (c) circumferential Cauchy stress $\sigma_{c}$.

\section{Finite element simulations}

In the previous section, bulge test was used to obtain stress and strain fields for a nonhomogeneous material. Stress were calculated without explicitly specifying a constitutive relation for the material. The aim of this section is to compare these results with usual finite element analysis using the classical Mooney-Rivlin hyperelastic constitutive equation. Stress-softening and hysteresis are not regarded, thus only the first loading behavior was investigated.

\subsection{Hyperelastic fitting using the Mooney-Rivlin hyperelastic model}

Assuming an incompressible isotropic hyperelastic material behavior, the two parameters Mooney-Rivlin model is expressed as

$$
\mathcal{W}\left(\bar{I}_{1}, \bar{I}_{2}\right)=C_{10}\left(\bar{I}_{1}-3\right)+C_{01}\left(\bar{I}_{2}-3\right)
$$


where $C_{10}$ and $C_{01}$ are material parameters that must be identified. $\bar{I}_{1}$ and $\bar{I}_{2}$ are the first and second strain invariants of the the isochoric elastic response. Due to its mathematical simplicity as well as its prediction accuracy in the range of moderately large strains, Mooney-Rivlin model has been widely employed in the description of the behavior of rubbery materials. It is known that different deformation modes are required to obtain the parameters that define the stressstrain relationship accurately. Uniaxial, planar and equibiaxial data acquired in Section 3 are simultaneously involved in a least square minimization in order to extract the sets of material parameters for each material phase. Table 2 summarizes the two sets of $C_{10}$ and $C_{01}$ parameters.

Table 2: Fitted parameters (in MPa) of the Mooney-Rivlin constitutive equation for soft and hard phases.

\begin{tabular}{c|c|c}
\hline Parameters & hard & soft \\
\hline$C_{10}$ & 0.35 & 0.18 \\
$C_{01}$ & 0.10 & 0.01 \\
\hline
\end{tabular}

Fig. 8 shows the stress-strain curves of the first loading experimental data and the MooneyRivlin model fitting for each deformation mode. The adopted fitting procedure allows a material model description that is valid for a general deformation state. As expected, the model shows a good agreement with uniaxial and planar tensile tests data up to $100 \%$ of strain, i.e., $\lambda=2.0$. Moreover, the Mooney-Rivlin model starts to fail to account strains larger than $\lambda=1.3$ for the equibiaxial tensile test, in particular for the hard phase. Nevertheless, as pointed out in [12], there are very few hyperelastic constitutive models able to simultaneously simulate both the multi-dimensional data with an unique set of material parameters.

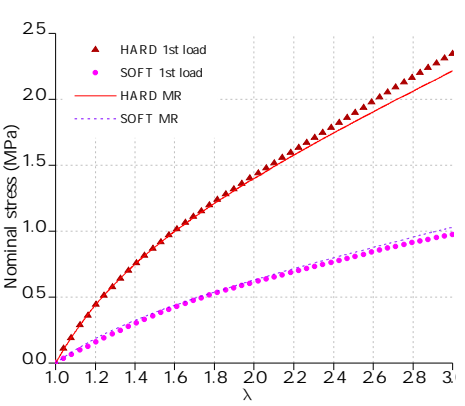

(a)

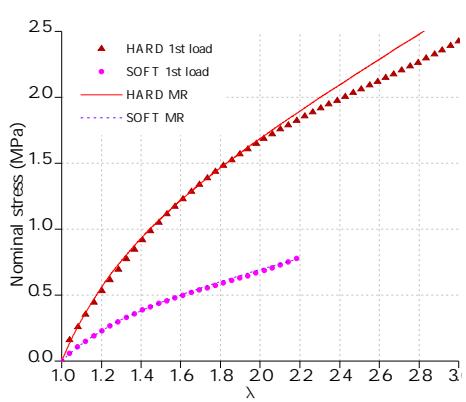

(b)

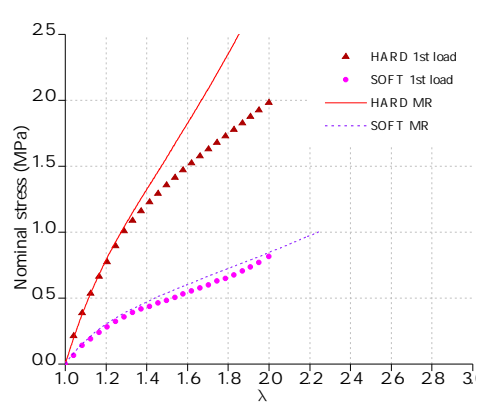

(c)

Figure 8: Experimental data for soft and hard phases and the hyperelastic fitting using the Mooney-Rivlin (MR) hyperelastic model: (a) Uniaxial (b) Planar and (c) equibiaxial tensile tests.

\subsection{Experimental and numerical comparison of bulge test results}

The non-homogeneous bulge test was simulated by an axisymmetric using Abaqus commercial finite element code. Continuum eight-node biquadratic hybrid fully integrated elements 
$(\mathrm{CAX} 8 \mathrm{H})$ were used. Based on the result of the mesh sensitivity study, the optimal global element size for membrane mesh was $h=35 \mu \mathrm{m}$. At the soft-hard interface, a non-adaptive h-refinement was used to improve mesh quality employing a mesh size gradient used only on the $R$ direction, resulting an element edge size about $h / 6$ over the interface neighborhood. Over the membrane thickness, 30 Gauss integration points were used.

Results of FEA using the Mooney-Rivlin model are superposed with the experimental fields (principal stretches and principal stresses) in Fig. 9. Two pressure levels were chosen to present the results: 6 and $25 \mathrm{kPa}$. All numerical predictions follow qualitatively the trends of the experimental data. It is possible to observe a discontinuity in the model response at $R=0.27$ even if the an h-refinement was used in this zone.

Considering the principal stresses plot in Figs.9c and 9d, numerical simulations do not correspond well with experimental ones in both load cases. This result can be related to the limitations of the Mooney-Rivlin model to fit complex stress-strain states at large strain levels.

Fig. 10a presents the FEA errors $\left(e_{m}, e_{c}\right)$ with respect to principal stresses over the bulge profile in both deformed configurations. Using a confidence interval of $95 \%$, the mean error are $\hat{e}_{m}=31 \%, \hat{e}_{c}=30 \%$ for the lower pressure level; and $\hat{e}_{m}=35 \%, \hat{e}_{m}=29 \%$ for the highest pressure level. Regardless of the soft-hard interface, stress discrepancies are independent of the deformation level. This fact is also observed in Fig. 10b where the deviations of the stress ratio $\sigma_{m} / \sigma_{c}$ are very close for both load levels. 


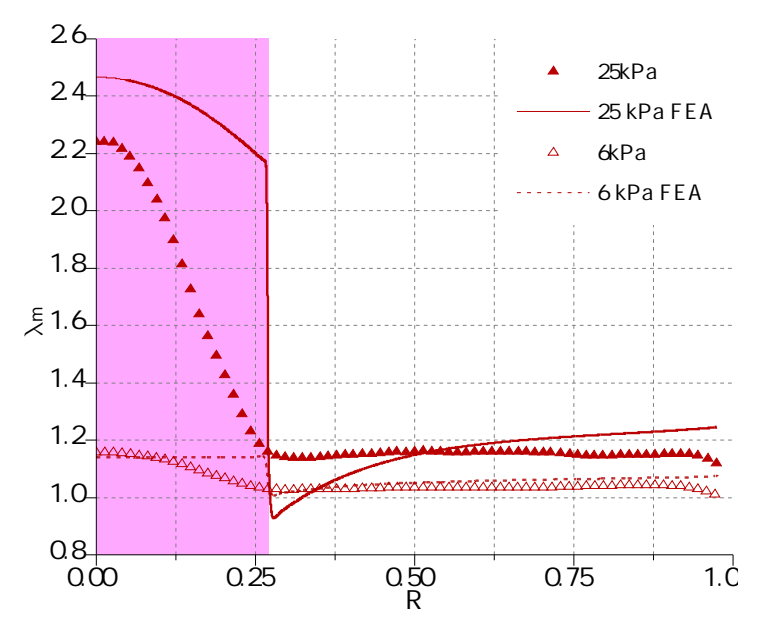

(a)

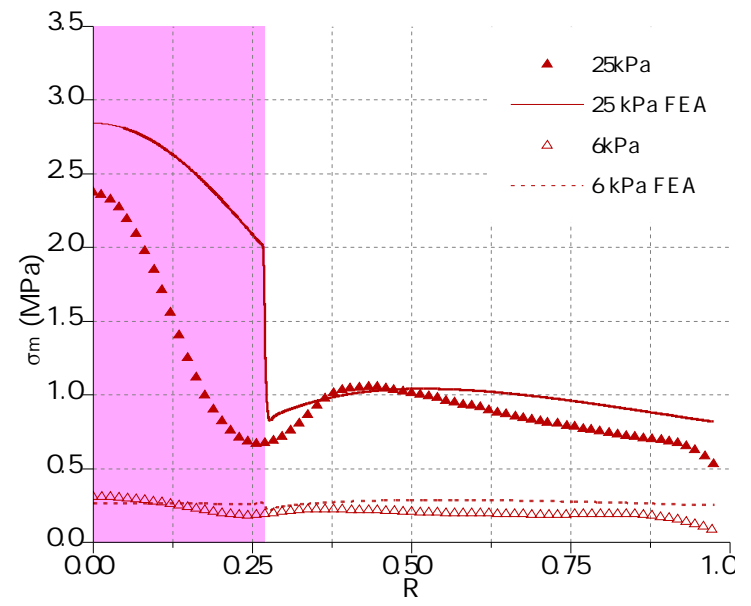

(c)

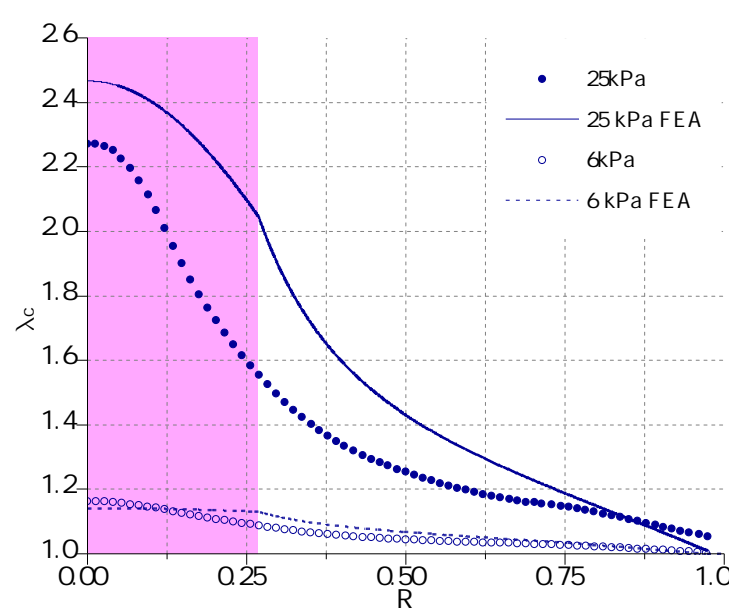

(b)

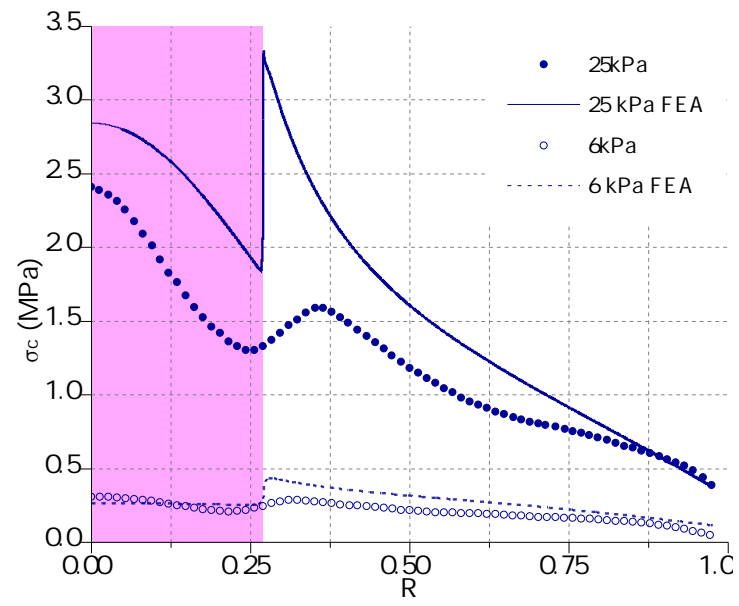

(d)

Figure 9: Principal stretches $\left(\lambda_{m}, \lambda_{c}\right)$ and Cauchy stress $\left(\sigma_{m}, \sigma_{c}\right)$ confronted with the finite element analysis (FEA), corresponding to $6 \mathrm{kPa}$ and $25 \mathrm{kPa}$ inflation states.
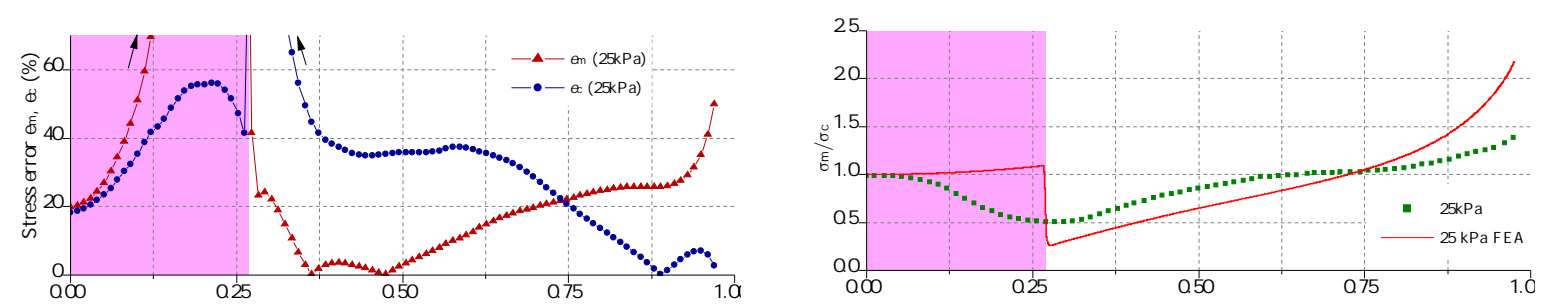

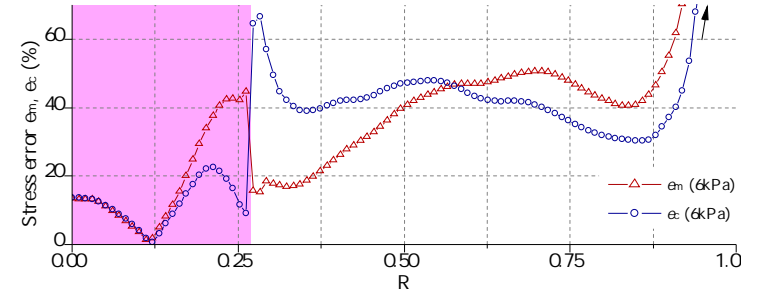

(a)

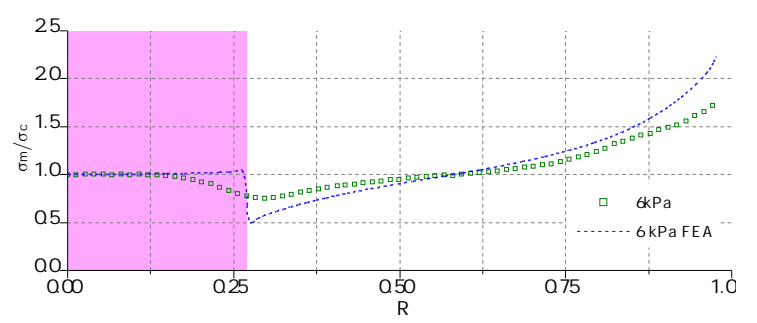

(b)

Figure 10: (a) FEA errors with respect to principal stresses in both deformed configurations; (b) Principal stress ratio $\left(\sigma_{m} / \sigma_{c}\right)$ confronted with the finite element results (FEA). 


\subsection{Modeling analysis}

Presented results show the limitations of the classical finite element method to tackle heterogeneous systems with moderate modulus mismatch across the material interface undergoing large strains, with an incompressible non-linear hyperelastic material behavior. Results reveals that a more sophisticate representation of the soft-hard interface must be taken into account by numerical modeling. For example, [13] proposed an extension of the generalized finite element method to tackle heterogeneous systems with non-linear hyperelastic materials. However, it must be recognized that outside the context of this study. Independently of the numerical treatment, a more detailed knowledge of the influence the material interface on the macroscopical mechanical behavior is necessary. For this, considering the Mooney-Rivlin model in Eq. 3 in terms of the principal stretches

$$
\begin{aligned}
\sigma_{m} & =2\left\{C_{10}\left[\left(\lambda_{m}\right)^{2}-\left(\frac{1}{\lambda_{m} \lambda_{c}}\right)^{2}\right]+C_{01}\left[\left(\frac{1}{\lambda_{m} \lambda_{c}}\right)^{2}-\left(\lambda_{m}\right)^{2}\right]\right\} \\
\sigma_{c} & =2\left\{C_{10}\left[\left(\lambda_{c}\right)^{2}-\left(\frac{1}{\lambda_{m} \lambda_{c}}\right)^{2}\right]+C_{01}\left[\left(\frac{1}{\lambda_{m} \lambda_{c}}\right)^{2}-\left(\lambda_{c}\right)^{2}\right]\right\} .
\end{aligned}
$$

Replacing the measured principal stretches in Eqs. 4 and 5 and the previous identified parameters $C_{10}$ and $C_{01}$ for the different phases, one obtains the local Mooney-Rivlin (MR) principal stresses calculated directly from the experimental local strain states. Regardless the interface zone, Figs. 11a and 11b shows a good agreement between the experimental (Exp) and Mooney-Rivlin (MR) stresses for both inflation states.

In other to determinate the macroscopic influence of the soft-to-hard transition the parameters $C_{10}$ and $C_{01}$ were evaluated using the Eqs. 4 and 5 using the the measured principal stretches and the experimental stresses obtained by Eqs. 1 and 2, using the different directions and different load levels experimental information. The same ratios $C_{10} / C_{01}$ of soft and hard phases from previous identification (Table 2) were kept. Thus, one obtains a description of the spatial distribution of these parameters, as presented in Fig. 12a. It is possible to observe that the soft-to-hard transition transition is almost symmetric with respect the position $R=0.27$ and the material parameters gradient extends over the $R=[0.21,0.38]$ interval.

The Mooney-Rivlin (MR) stresses in Eqs. 4 and 5 were recalculated, but now using the functions $C_{10}(R)$ and $C_{01}(R)$, fitted on experimental results using a sigmoid function. Results 
are plotted in Figs.11c and 11d and a good agreement is observed even in the soft-to-hard transition. Fig. 12b presents the distribution of errors with respect to principal stresses, when a material parameter gradient is used to describe the soft-to-hard transition. Using a confidence interval of $95 \%$, the mean error are $\hat{e}_{m}=7 \%, \hat{e}_{c}=6 \%$ for the lower pressure level; and $\hat{e}_{m}=6 \%$, $\hat{e}_{m}=3 \%$ for the highest pressure level.

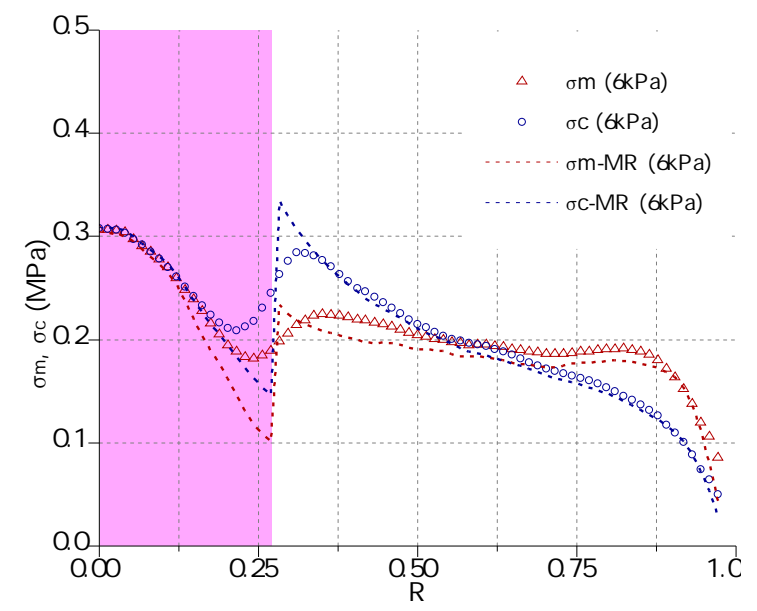

(a)

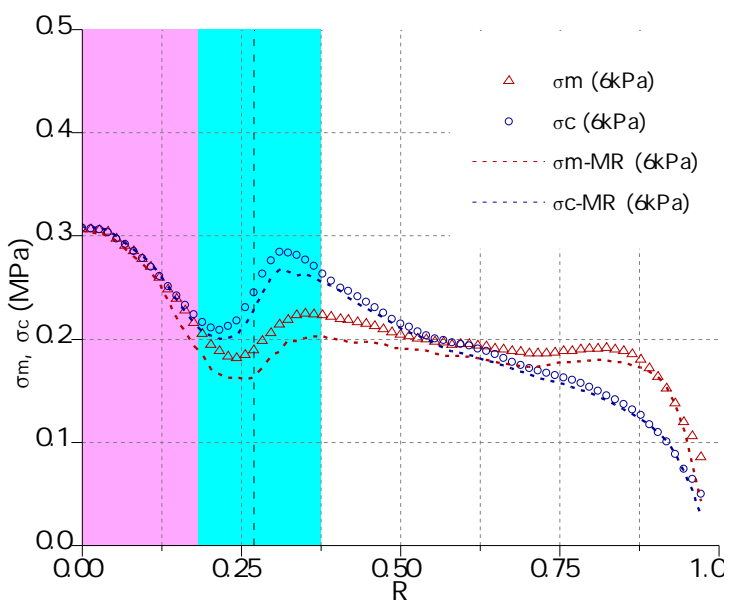

(c)

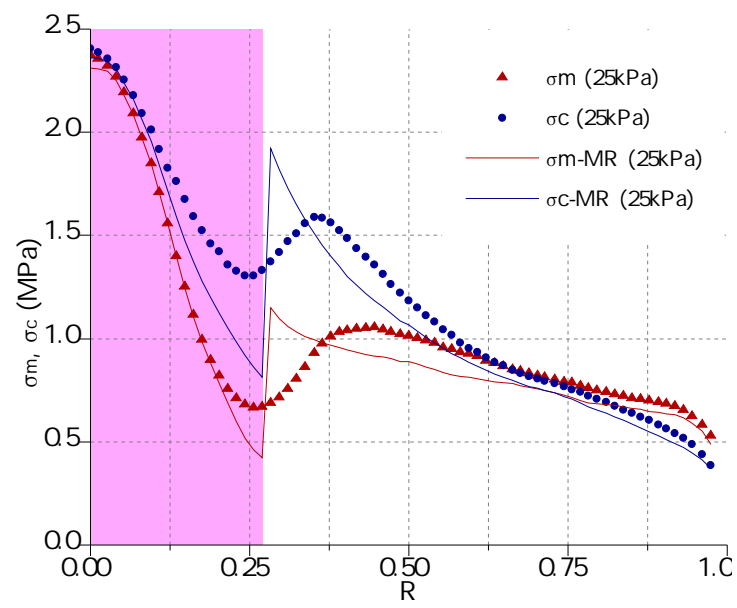

(b)

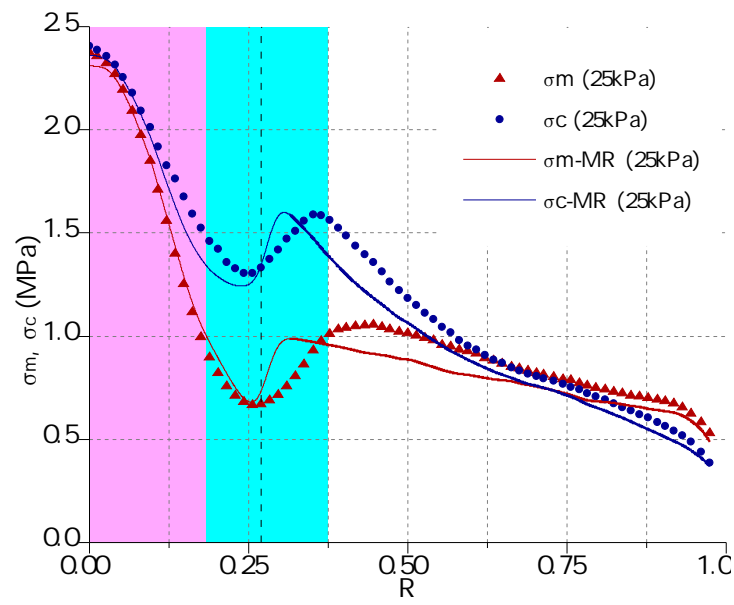

(d)

Figure 11: Distributions of experimental (Exp) and Mooney-Rivlin (MR) calculated directly from the experimental local strain states corresponding to $6 \mathrm{kPa}$ and $25 \mathrm{kPa}$ inflation states. (a) and (b): principal stresses $\left(\sigma_{m}, \sigma_{c}\right)$ obtained using a single parameter set; (b) and (c) principal stresses $\left(\sigma_{m}, \sigma_{c}\right)$ using a material parameters gradient over the $R=[0.21,0.38]$ interval. 


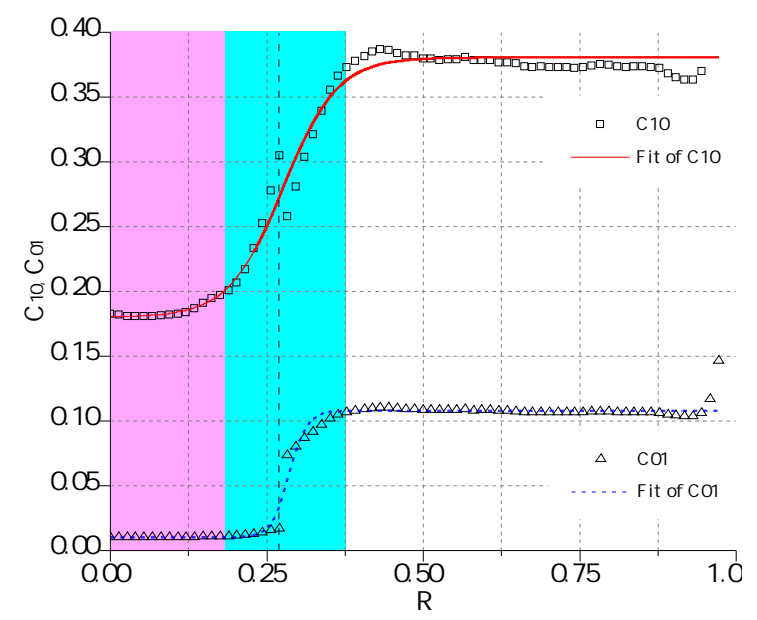

(a)
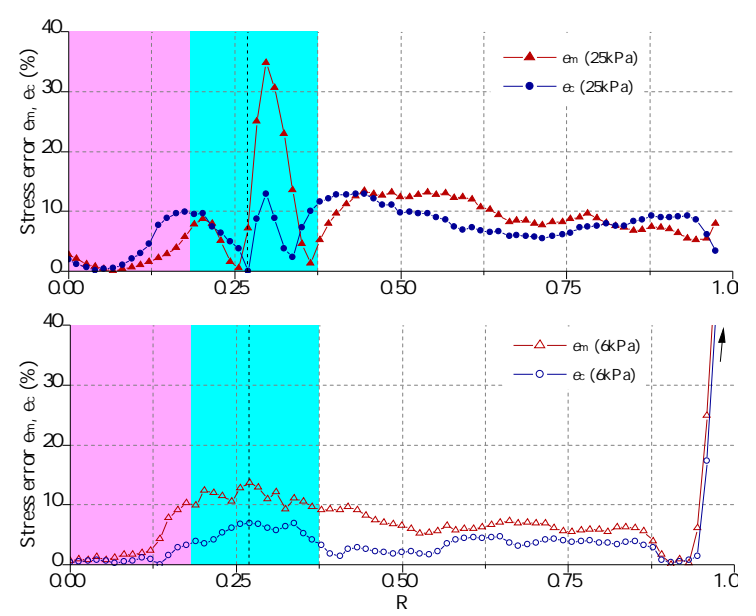

(b)

Figure 12: (a) Mooney-Rivlin parameters gradient obtained using the the experimental local stress-strain states. (b) Errors with respect to principal stresses in both deformed configurations using the Mooney-Rivlin with a material parameters gradient over the $R=[0.21,0.38]$ interval.

Finally, keeping in mind the experimental spatial resolution of $15 \mu \mathrm{m}$, the phase transition is estimated to be about $3.15 \mathrm{~mm}$, i.e., $17 \%$ total sample radius. Within the tested loading range, the size of soft-to-hard transition can be assumed independent of the stress-strain level.

\section{Conclusions}

Results show the mechanical behavior of photosensitive silicone membranes with a variable set of mechanical properties within the same material. With a reversible in-plane stretchability up to $200 \%$, the soft-to-hard transition was expressed by a factor 3.57 in the Young's modulus within a single continuous silicone membrane combined with a mean stress factor about 2.5 times.

The approach was tested using the bulge test and the presented results using a bimaterial are distinct from previous investigations of the classic circular homogeneous membrane inflation problem. The mechanical response of the soft-hard interface was observed by inflections on the principal curvatures fields along the meridional and circumferential directions. Analysis of the stress distribution throughout the meridional-section of the membrane revealed low stress peaks at soft-to-hard transition. The results demonstrate that under high strains levels no macroscopic damage was detected. The local cross-linking control eliminates the interfaces between different materials, leading to heterogeneous membrane with efficient stress transfer throughout the structure. 
The numerical investigation provided information on the respective contributions of each material phase on its effective behavior under the inflation. Therefore, presented results show the limitations of the classical finite element method to tackle heterogeneous systems with moderate modulus mismatch across the material interface undergoing large strains, with an incompressible non-linear hyperelastic material behavior. Using the experimental local stress-strains values, it was possible to characterize the macroscopic influence of the soft-to-hard interface with spatial resolution of $15 \mu \mathrm{m}$. Later, a more sophisticated numerical strategy can be used to describe the soft-hard interface and then the graded membrane global behavior, based on the presented results. Further work to create, test and optimize more complex architectures, is ongoing using the experimental approaches described in the present paper.

\section{Acknowledgment}

The authors wish to acknowledge the financial support of the French ANR research program SAMBA: Silicone Architectured Membranes for Biomedical Applications (Blanc SIMI 9 2012). We thank Laurent Chazeau, Renaud Rinaldi and Franois Ganachaud for fruitful discussions.

\section{References}

[1] Y. Brechet, J. Embury Architectured materials: Expanding materials space. Scripta Mater, vol. 68, pp. 1-3, 2013.

[2] F. Zuo, Y. Mao, X. Li, C. Burger, B.S. Hsiao. Effects of block architecture on structure and mechanical properties of olefin block copolymers under uniaxial deformation. Macromolecules, vol. 44, pp. 3670-3673, 2011.

[3] L. Bailly, M. Toungara, L. Orgéas, E. Bertrand, V. Deplano, C. Geindreau. In-plane mechanics of soft architectured fibre-reinforced silicone rubber membranes. J Mech Behav Biomed Mater, vol. 40, pp. 339-353, 2014.

[4] T. Rey, J-B. Le Cam, G. Chagnon, D. Favier, M. Rebouah, F. Razan, E. Robin, P. Didier, L. Heller., S. Faure, K. Janouchova. An original architectured NiTi silicone rubber structure for biomedical applications. Mater Sci Eng C, vol. 45, pp. 184-190, 2014.

[5] L. Meunier, G. Chagnon, D. Favier, L. Orgéas. Anisotropic large deformation of geometrically architectured unfilled silicone membranes. Eur J Mech A-Solid, vol. 50, pp. 87-99, 2015.

[6] M. Rebouah, G. Chagnon, D. Favier. Development and modeling of filled silicone architectured membranes. Meccanica, vol. 50, pp. 11-24, 2015. 
[7] M.A. Sutton, J-J. Orteu, H.W. Schreier. Image correlation for shape, motion and deformation measurements. 1st ed., pp. 88-99, Springer, New York, 2009.

[8] R. Hill. A theory of the plastic bulging of a metal diaphragm by lateral pressure. Philos Mag, vol. 41, pp. $1133-1142,1950$.

[9] A.S. Wineman. Large axisymmetric inflation of a nonlinear viscoelastic membrane by lateral pressure. Trans Soc Rheol, vol. 20, pp. 203-225, 1976.

[10] J. Humphrey. Computer methods in membrane biomechanics. Comput Meth Biomech Biomed Eng, vol. 1, pp. 171-210, 1998.

[11] G. Machado, D. Favier, G. Chagnon. Membrane curvatures and stress-strain full fields of axisymmetric bulge tests from 3D-DIC measurements. Theory and validation on virtual and experimental results. Exp Mech, vol. 52 , pp. 865-880, 2012.

[12] G. Marckmann, E. Verron. Comparison of hyperelastic models for rubberlike materials. Rub Chem Tech, vol. 79, pp. 835-858, 2006.

[13] K. Srinivasan, K. Matouš, P. Geubelle. Generalized finite element method for modeling nearly incompressible bimaterial hyperelastic solids. Comput Meth Appl Mech Eng, vol. 197, pp. 4882-4893, 2008. 